\title{
Enhancing Project Management Efficiency using Lean Concepts
}

\author{
Mr. Harsha N. ${ }^{1}$ and Mr. S. Nagabhushan ${ }^{2}$

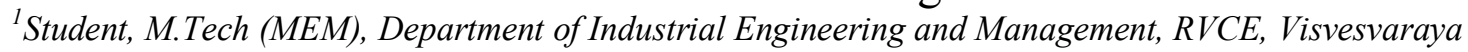 \\ Technological University, Bangalore, Karnataka, India \\ ${ }^{3}$ Research Scholar, Visvesvaraya Technological University, Bangalore, Karnataka, India
}

\begin{abstract}
One of the increasing and most significant concerns with projects is that, projects are behind schedule, over budget and show unsatisfactory performance in terms of quality and customer satisfaction. Lean Thinking is important because it can reduce error rates to maximum extent. The evidence from Toyota (Japan) and other companies who have successfully implemented the Toyota Production System (TPS) or Lean Principles confirms this. It also significantly reduces the time taken to deliver new products while substantially reducing cost. This paper first introduces the general overview about Project Management and the Lean Concepts. After that, this paper presents the literature survey and then compares the traditional Project Management, the advantages or opportunities of introducing Lean Principles into Project Management with a Case study.
\end{abstract}

Keywords - Lean Project Management, Project Management, Toyota Production System

\section{Introduction}

Today organizations face challenges in the form of intense international competition, rapid technology evolution, maturing customer expectations and quality demands (King, 1987). In such circumstances, effective and efficient management is among the primary ways in which organizations adapt for their existence. Lean project management is the comprehensive adaption of lean concepts like Lean Construction, Lean Manufacturing and Lean Thinking into a Project Management context. Lean Project Management has many ideas in common with lean concepts. However, the main principle of lean project management is delivering more value with less waste in a project context. Lean Project Management has many techniques that can be applied to projects and one of the main methods is standardization. Standardization is the process of developing and implementing technical standards. The goals of standardization can be of help with independence of single suppliers, compatibility, interoperability, safety, repeatability or quality.

\section{Lean Project Management}

In recent years, Project Management has become a central way for undertaking business activities in an effective way (Whittington, et al., 1999). The activities that are often undertaken in the form of projects belong to different industries, such as services, construction, administration, management, product development, etc. However, several of these projects are condemned to be delayed and overrun cost, which is often considered as normal. This passive acceptance that projects may not deliver on time or within allocated budget has great effects on the overall business of organizations. Delay in projects fairly means a delay in product introduction, certainly missing market opportunity and forefront of technology, which seriously affects company's superiority in market. Further, it is also identified that projects often show unsatisfactory performance with respect to productivity and quality (Atkinson, 1999; Winch, 1996).

In order to address the problems above, a new perspective to project management may lead to new insights that can improve project performance. In the field of manufacturing, for instance, tremendous improvements in productivity and quality have been made by the implementation of lean. It could be interesting to investigate to what extent (part of) the practices connected to lean could be useful in project management. However, project management is a field which is relatively different from manufacturing management. Projects are temporary and unique, and are performed by teams with clearly defined individual roles (Tonnquist, 2008). In contrast, manufacturing is based on ongoing and repetitive activities conducted by production personnel. It is also a common practice to initiate project for unique and creative tasks, for example, product development activities. Product development projects are based on a high degree of ambiguity, uncertainty and are also complex in nature, especially regarding radical endeavors (O'Connor, 2008; Trott, 2008). Generally, for these activities a higher degree of creativity is required, which is connected to more slack and freedom in work activities (Millson, et al., 1992). However, there are also some common characteristics between manufacturing and projects as both are performed by people, are constrained by limited resources, both are planned, executed and controlled, but maybe to different degrees. 
In the last few decades the manufacturing industry successfully improved productivity, by developing new methods and ways of working. Especially lean manufacturing became popular and is recognized as a main reason of success, efficiency and high productivity (Mohanty, et al., 2006; Cusumano, 1992). The principles of lean manufacturing are now well established and are widely used not only in automotive industry, but also in other industries, and in different contexts, like services.

The aim of this paper is to systematically review various research works that has been carried out in the area of lean project management consisting of the lean principles to diversified projects to enhance efficiency and reduce waste.

\section{Literature Review}

The paper 'Lean approach to Project Management' by Eric Gabriel [5] illustrates case studies that developed the concept of lean project management, which recognizes that the system and philosophy is now better understood by participants, who themselves provide and contribute to the project control mechanisms.

Qi Shenjun [6] through the paper 'Study on Integration and Management System of Schedule in Large Complex Construction Engineering Projects Based on Lean Construction' presents the basic theory of Lean Construction and multistage plans of construction engineering project, Integration and Management System of Schedule in Large Complex Construction Engineering Projects (IMSSLCCEP) was proposed. It was studied from the data flow, hierarchy and functional model. Finally the cases of applying of IMSSLCCEP were studied, which indicated that IMSSLCCEP was an effective way of reduction for large complex construction engineering schedule management from the whole angle.

Li Juanfang and Liu Xing in 'Application of Lean Construction in Quality Management of Engineering Projects' [7] paper presents the general overview about today's project quality management in China, then explains lean construction theory. After that, compares with the traditional quality management elaborating the similarity and difference. At last, but not the least, discusses about four practical factors for providing a way to be possibly used in the industry.

Basit Aziz, with his paper 'Improving Project Management with Lean Thinking' [8] gives a general overview on Project Management, Lean Manufacturing principles and the opportunities of Lean Project Management process.

The Lean Innovation System represents the systematic interpretation of Lean Thinking principles regarding to product or process innovation and development. One core element of Lean Innovation is the Value System which is the basis for the value stream design in innovation and development projects. The Value System defines structures and priorities 'values' adaptively for one specific innovation project. The values are defined by all relevant stakeholders in the innovation and development process, like external and internal customers, considering company's strategy and culture. It represents the basis for a consequent value oriented alignment of project and processes in R\&D.

Gunther Schuh, through the paper 'Lean Innovation: Introducing Value Systems to Product Development' [9] introduces Lean Innovation and the core findings of the recent survey 'Lean Innovation' of the Laboratory for Machine Tools and Production Engineering WZL at RWTH Aachen University and focuses on the Value System, describes its elements and shows how to use and benefit from the Value System towards a powerful Lean Innovation.

Glenn Ballard and Gregory A. Howell, in 'Lean Project Management' [10], states that Projects are temporary production systems. When those systems are structured to deliver the product while maximizing value and minimizing waste, they are said to be 'lean' projects. Lean project management differs from traditional project management not only in the goals it pursues, but also in the structure of its phases, the relationship between phases and the participants in each phase. This paper also presents a model of lean project management and contrasts lean and traditional approaches. Four tools or interventions are presented as illustrations of lean concepts in action.

Willie Tan with his paper 'Managing Lean Projects: Understanding the Structures of Lean Production' [11] gives an assessment on problems with lean production (LP) in the wake of recent worldwide recalls of millions of cars by automakers at the forefront of LP. The paper defines LP more broadly than the literature to include the structures of culture and political economy. It uses Toyota as a case study to identify the structural weaknesses of the entire lean production system beyond the shop floor. The main findings are, firstly, that the Toyota system is not well understood, and often misunderstood, with respect to its possible political - economic and social conditions of existence. This implies that the system cannot be easily replicated elsewhere. Secondly, changing structural conditions, particularly in the labor market and industrial system, have encouraged Toyota to introduce slower lines of production for older and female workers, use buffers to overcome more frequent production halts, and reduce the design cycle. These findings are of value to project managers who wish to obtain a deeper insight into the structural problems of LP and project management. 


\section{Case Study}

A case of 'Lean Software Management: BBC' by Peter Middleton and David Joyce has been studied. This case study examines how the lean ideas behind the Toyota production system can be applied to software project management. It is a detailed investigation of the performance of a nine - person software development team employed by BBC - Worldwide based in London.

The data collected in 2009 involved direct observations of the development team, the kanban boards, the daily stand - up meetings, semi structured interviews with a wide variety of staff and statistical analysis. The evidence shows that over the 12 - month period, lead time to deliver software improved by $37 \%$, consistency of delivery rose by $47 \%$ and defects reported by customers fell by $24 \%$. The significance of this work is showing that the use of lean methods including visual management, team - based problem solving, smaller batch sizes, and statistical process control can improve software development. It also summarizes key differences between agile and lean approaches to software development.

The conclusion is that the performance of the software development team was improved by adopting a lean approach. The faster delivery with a focus on creating the highest value to the customer also reduced both technical and market risks. The drawbacks are that it may not fit well with existing corporate standards.

\section{CONCLUSION}

This paper oversees an alternative methodology to Project Management that has been discussed and termed Lean Project Management. This methodology is derived from the combining of Lean Production with Management of Projects. The approach contributes to project management performance by focusing on the effectiveness and efficiency of delivering value. This methodology utilizes elements of both Lean Production and the Project Management. Lean production enhances the conventional production methodology by emphasizing the efficient provision of value. It does this by introducing flow management and the management of value on an equal par to input, conversion and output management.

Further research work has to be done in the areas of Lean Project Management, in order to utilize the available resource in optimistic way and gain enhanced efficiency in managing the projects.

\section{REFERENCES}

[1] Womack J., Jones D. and Roos T. (1990a), “The Origins of Lean Production”, Machine That Changed the World, MacMillanCanada, pp. 26-33

[2] Liker, K. Jeffrey. 'The Toyota Way; 14 management principles from the world's greatest manufacturer, McGraw-Hill 2004.

[3] Atkinson, R., 1999. Project management: Cost, time and quality, two best guesses and a phenomenon; It's time to accept other success criteria. International Journal of Project Management, 17(6), pp. 337-342.

[4] Womack, James. P., Jones, Daniel. T., (2003) Lean Thinking: Banish waste and create wealth in your corporation, 2nd ed., Simon \& Schuster UK Ltd.

[5] Eric Gabriel, 'Lean approach to Project Management' International Journal of Project Management Vol. 15, No. 4, pp. 205-209, 1997

[6] Qi Shenjun, Ding Lieyun, Luo Hanbin, 'Study on Integration and Management System of Schedule in Large Complex Construction Engineering Projects Based on Lean Construction'. 978-0-7695-4115-0/10 IEEE 2010.

[7] Li Juanfang and Liu Xing 'Application of Lean Construction in Quality Management of Engineering Projects', 978-1-4244-86946/11, IEEE 2011.

[8] Basit Aziz, 'Improving Project Management with Lean Thinking' INN Division of Project, Innovation and Entrepreneurship (PIE) LIU-IEI-TEK-A--12/01272-SE.

[9] Günther Schuh, Michael Lenders, Solveigh Hieber, 'Lean Innovation: Introducing Value Systems to Product Development' PICMET (2008) South Africa.

[10] Glenn Ballard and Gregory A.Howell, 'Lean project management' Building Research \& Information ISSN 0961-3218 print/4SSN 1466-4321 online \# 2003 Taylor \& Francis Ltd.

[11] Willie TAN 'Managing lean projects: understanding the structures of lean production' The International Journal of Construction Management (2011) Vol. 11 No. 3, 67-78. 